\title{
Loss of non-Gaussianity for damped photon-subtracted thermal states
}

\author{
Iulia Ghiu ${ }^{1}$, Paulina Marian ${ }^{1,2}$, and Tudor A. Marian ${ }^{1}$ \\ ${ }^{1}$ Centre for Advanced Quantum Physics, Department of Physics, University of \\ Bucharest, R-077125 Bucharest-Măgurele, Romania \\ 2 Department of Physical Chemistry, University of Bucharest, Boulevard Regina \\ Elisabeta 4-12, R-030018 Bucharest, Romania \\ E-mail: iulia.ghiu@g.unibuc.ro \\ paulina.marian@g.unibuc.ro \\ tudor.marian@g. unibuc.ro
}

\begin{abstract}
We investigate non-Gaussianity properties for a set of classical one-mode states obtained by subtracting photons from a thermal state. Three distance-type degrees of non-Gaussianity used for these states are shown to have a monotonic behaviour with respect to their mean photon number. Decaying of their nonGaussianity under damping is found to be consistently described by the distancetype measures considered here. We also compare the dissipative evolution of nonGaussianity when starting from $M$-photon-subtracted and $M$-photon-added thermal states.
\end{abstract}

PACS numbers: 42.50.Ar; 03.65.Yz; 03.67.-a 


\section{Introduction}

In quantum optics, non-Gaussian states were studied in connection with some nonclassical properties such as photon antibunching and quadrature or amplitude-squared squeezing. A survey on non-classicality defined as the non-existence of the GlauberSudarshan $P$ representation as a genuine probability density can be found in Ref. [1]. Interest in the non-Gaussian states has then renewed in quantum information processing due to their efficiency in some quantum protocols [2, 3. In general, their usefulness was connected to certain non-classicality properties detected by negative values of the Wigner function. However, it has recently been realized that quantum states with nonnegative (Gaussian or non-Gaussian) Wigner functions can be efficiently simulated on a classical computer [4, 5]. It appears that non-Gaussianity of a quantum state is a useful feature in quantum information processing, regardless of being non-classical or classical according to the concepts of quantum optics. To quantify this property as a resource, some non-Gaussianity measures were recently defined as distances between the given state $\hat{\rho}$ and its associate Gaussian state $\hat{\tau}_{G}$. Here $\hat{\tau}_{G}$ is the unique Gaussian state having the same mean displacement and covariance matrix as $\hat{\rho}[6,7$, , 8, 9, 10]. In Refs. [6, 7, 8], Genoni et al. used the Hilbert-Schmidt metric and the relative entropy as distances and gave a comprehensive discussion of the general properties of non-Gaussianity degrees for large sets of one-mode, two-mode, and multimode states. Later, in Refs. [9, 10] a degree of non-Gaussianity based on the Bures metric was similarly introduced. We stress now that all the distance-type measures considered so far used the same state $\hat{\tau}_{\mathrm{G}}$ as a reference Gaussian state in evaluating non-Gaussianity. It was only very recently that two of us succeeded to prove that the relative entropy of any $N$-mode state to its associate Gaussian one $\hat{\tau}_{\mathrm{G}}$ is an exact distance-type measure of non-Gaussianity [1].

All these measures were already employed to evaluate non-Gaussianity degrees in some interesting experiments. In the experiment reported in Ref. [12] the relativeentropy measure was used for single-photon-added coherent states, while in Ref. [13] the same degree was evaluated in an experiment with multiple-photon subtraction from a thermal state. The three above-mentioned degrees of non-Gaussianity were determined and compared in some recent experiments on phase-averaged coherent states [14, 15].

The present work parallels some of our recent findings on the non-Gaussianity and its decay in contact with a thermal reservoir for an interesting class of Fock-diagonal one-mode states: the photon-added thermal states [9, 10]. Here we intend to compare the three distance-type amounts of non-Gaussianity during the damping of two different excitations on a single-mode thermal state: an $M$-photon-added thermal state (PATS) and an $M$-photon-subtracted thermal state (PSTS).

The plan of our paper is as follows. In Section 2 we recall some statistical properties of a PSTS. In Section 3 the three usual degrees of non-Gaussianity for a Fock-diagonal one-mode state are recapitulated. Then we derive an analytic expression of the HilbertSchmidt measure of non-Gaussianity for a PSTS. Plots of the entropic and Bures amounts of non-Gaussianity are shown to be in agreement with the Hilbert-Schmidt 
measure. Section 4 examines the evolution of a PSTS due to the interaction of the field mode with a thermal reservoir, which is governed by the quantum optical master equation. We finally compare the decay of non-Gaussianity for pairs of states, PSTSs and PATSs, having the same thermal mean occupancy $\bar{n}$, as well as the same number $M$ of subtracted and, respectively, added photons. Our concluding remarks are presented in Section 5.

\section{Multiple-photon-subtracted thermal states}

It is known that excitations on a Gaussian state $\hat{\rho}_{G}$ of the type $\hat{\rho} \sim\left(\hat{a}^{\dagger}\right)^{k} \hat{a}^{l} \hat{\rho}_{G}\left(\hat{a}^{\dagger}\right)^{l} \hat{a}^{k}$ lead to non-Gaussian states [16, 17, 18, 2, 19]. Here $\hat{a}$ and $\hat{a}^{\dagger}$ are the amplitude operators of the field mode. Interesting experiments on such states were recently conducted to enlighten their fundamental features [20, 21, 22, 23, 13]. Addition of photons to any classical Gaussian state, in particular to a coherent or a thermal one, generates a nonGaussian output which is no longer classical [16]. In Refs. [9, 10] we have recently studied the non-Gaussianity of $M$-photon-added thermal states and investigated their behaviour under damping. Their non-classicality was marked by the negativity of both Wigner and $P$ functions surviving to some extent under damping as well. On the contrary, an $M$-photon-subtracted Gaussian state can be either classical or non-classical, depending on the input state $\hat{\rho}_{G}[24,25,26]$. For instance, subtraction of photons from a coherent or a thermal state provides a classical non-Gaussian output [24, 27]. Therefore, the PSTSs are an interesting example of classical non-Gaussian Fock-diagonal states. Measurement of their photon statistics by means of photon-number-resolved detection was recently reported [28]. In this section we recall some of their statistical properties.

We thus consider an arbitrary single-mode PSTS:

$$
\hat{\rho}_{M}^{\mathrm{sub}}(\bar{n})=\frac{1}{M !(\bar{n})^{M}} \hat{a}^{M} \hat{\rho}_{\mathrm{T}}(\bar{n})\left(\hat{a}^{\dagger}\right)^{M}, \quad(M=1,2,3, \ldots) .
$$

Here $M$ is the number of photons extracted from the mode and $\hat{\rho}_{\mathrm{T}}(\bar{n})$ is a thermal state whose mean number of photons is $\bar{n}>0$ :

$$
\hat{\rho}_{\mathrm{T}}(\bar{n})=(1-x) \sum_{n=0}^{\infty} x^{n}|n\rangle\langle n| \quad \text { with } \quad x:=\frac{\bar{n}}{\bar{n}+1}>0 .
$$

A PSTS (2.1) is Fock-diagonal, with the photon-number probabilities

$$
p_{n}^{\mathrm{sub}}:=\left[\rho_{M}^{\mathrm{sub}}(\bar{n})\right]_{n n}=\left(\begin{array}{c}
n+M \\
M
\end{array}\right)(1-x)^{M+1} x^{n}, \quad(n=0,1,2,3, \ldots) .
$$

This is actually a negative binomial distribution [29] with the stopping parameter $r=M+1$. Its generating function is

$$
\mathcal{G}_{M}^{\mathrm{sub}}(\bar{n}, v):=\sum_{n=0}^{\infty} p_{n}^{\mathrm{sub}} v^{n}=\left(\frac{1-x}{1-x v}\right)^{M+1}, \quad(-1 \leqq v \leqq 1) .
$$


Accordingly, the mean number of photons in the PSTS (2.1),

$$
\langle\hat{n}\rangle:=\sum_{n=0}^{\infty} n p_{n}^{\mathrm{sub}}=\left[\frac{\partial}{\partial v} \mathcal{G}_{M}^{\mathrm{sub}}(\bar{n}, v)\right]_{v=1},
$$

has the expression:

$$
\langle\hat{n}\rangle=(M+1) \bar{n} .
$$

It is therefore proportional to the thermal mean occupancy $\bar{n}$ and, rather counterintuitively, increases with the number $M$ of extracted photons. Note that the purity of a PSTS (2.1),

$$
\operatorname{Tr}\left\{\left[\hat{\rho}_{M}^{\mathrm{sub}}(\bar{n})\right]^{2}\right\}=\sum_{n=0}^{\infty}\left(p_{n}^{\mathrm{sub}}\right)^{2}=(1-x)^{2(M+1)}{ }_{2} F_{1}\left(M+1, M+1 ; 1 ; x^{2}\right),
$$

where ${ }_{2} F_{1}$ is a Gauss hypergeometric function (A.1), coincides with that of the PATS $\hat{\rho}_{M}^{\text {add }}(\bar{n})$, which was written in Ref. [9]:

$$
\operatorname{Tr}\left\{\left[\hat{\rho}_{M}^{\mathrm{sub}}(\bar{n})\right]^{2}\right\}=\operatorname{Tr}\left\{\left[\hat{\rho}_{M}^{\mathrm{add}}(\bar{n})\right]^{2}\right\}, \quad(M=1,2,3, \ldots) .
$$

An equivalent form of Eq. (2.7) in terms of a Legendre polynomial $\mathcal{P}_{M}$ can be obtained by using Eqs. (A.2) and (A.3):

$$
\operatorname{Tr}\left\{\left[\hat{\rho}_{M}^{\mathrm{sub}}(\bar{n})\right]^{2}\right\}=\left(\frac{1-x}{1+x}\right)^{M+1} \mathcal{P}_{M}\left(1+\frac{2 x^{2}}{1-x^{2}}\right) .
$$

\section{Non-Gaussianity of a photon-subtracted thermal state}

We do not insist on the general properties of the distance-type degrees of nonGaussianity introduced in Refs. [6, 7, 9]. We just recall the three degrees of nonGaussianity written for a Fock-diagonal state $\hat{\rho}$ (our case in the following),

$$
\hat{\rho}=\sum_{n=0}^{\infty} p_{n}|n\rangle\langle n| \quad \text { with } \quad \sum_{n=0}^{\infty} p_{n}=1 .
$$

In this case, the associate Gaussian state is a thermal one with the same mean photon occupancy, $\langle\hat{n}\rangle=\sum_{n=0}^{\infty} n p_{n}$ :

$$
\hat{\tau}_{\mathrm{G}}=\sum_{n=0}^{\infty} s_{n}|n\rangle\langle n| \quad \text { with } \quad s_{n}:=\frac{1}{\langle\hat{n}\rangle+1} \sigma^{n}, \quad \sigma:=\frac{\langle\hat{n}\rangle}{\langle\hat{n}\rangle+1} .
$$

The Hilbert-Schmidt and entropic amounts of non-Gaussianity were written in Refs. [6, 7, 9] as:

$$
\begin{aligned}
\delta_{\mathrm{HS}}[\hat{\rho}]= & \frac{1}{2}\left[1+\frac{\sum_{n}\left(s_{n}^{2}-2 s_{n} p_{n}\right)}{\sum_{n} p_{n}^{2}}\right] \\
& =\frac{1}{2}+\frac{1}{2 \operatorname{Tr}\left(\hat{\rho}^{2}\right)}\left[\frac{1}{2\langle\hat{n}\rangle+1}-\frac{2}{\langle\hat{n}\rangle+1} \mathcal{G}_{\hat{\rho}}(\sigma)\right],
\end{aligned}
$$

and, respectively,

$$
\delta_{\mathrm{RE}}[\hat{\rho}]=\sum_{n=0}^{\infty} p_{n} \ln p_{n}+(\langle\hat{n}\rangle+1) \ln (\langle\hat{n}\rangle+1)-\langle\hat{n}\rangle \ln (\langle\hat{n}\rangle) .
$$


Here we have used the purity of the thermal state $\hat{\tau}_{G}$ arising from Eq. (3.2), while $\mathcal{G}_{\hat{\rho}}(y):=\sum_{n=0}^{\infty} p_{n} y^{n}$ is the generating function of the photon-number distribution in the given state $\hat{\rho}$.

The third measure of interest was introduced in terms of the Bures distance between the state $\hat{\rho}$ and its associate Gaussian state $\hat{\tau}_{\mathrm{G}}$ [9, 10. In the case of a Fock-diagonal state, we notice the commutation relation $\left[\hat{\rho}, \hat{\tau}_{\mathrm{G}}\right]=\hat{0}$, which implies the simpler formula:

$$
\delta_{\mathrm{F}}[\hat{\rho}]=1-\sum_{n=0}^{\infty} \sqrt{p_{n} s_{n}} .
$$

The Hilbert-Schmidt degree of non-Gaussianity, Eq. (3.3), can readily be evaluated for any PSTS. First, we employ Eqs. (2.6) and (3.2) to write the generating function (2.4) for $v=\sigma$ :

$$
\mathcal{G}_{M}^{\mathrm{sub}}(\bar{n}, \sigma)=\left[\frac{(M+1) \bar{n}+1}{(M+2) \bar{n}+1}\right]^{M+1} .
$$

Then, by replacing Eqs. (2.9) and (3.6) into Eq. (3.3), one finds the formula:

$$
\begin{aligned}
\delta_{\mathrm{HS}}\left[\hat{\rho}_{M}^{\mathrm{sub}}(\bar{n})\right]= & \frac{1}{2}+\frac{(2 \bar{n}+1)^{M}}{2 \mathcal{P}_{M}\left(1+\frac{2 \bar{n}^{2}}{2 \bar{n}+1}\right)}\left\{\frac{1}{1+\frac{2 M \bar{n}}{2 \bar{n}+1}}\right. \\
& \left.-\frac{2}{1+\frac{M \bar{n}}{2 \bar{n}+1}}\left[\frac{(M+1) \bar{n}+1}{(M+2) \bar{n}+1}\right]^{M}\right\} .
\end{aligned}
$$

Plots of the Hilbert-Schmidt measure (3.7) versus the number $M$ of subtracted photons at some values of the thermal mean occupancies are shown in Figure 1 (right). In Figure 2 (right) we keep constant the value of $M$ and give the dependence of $\delta_{\mathrm{HS}}$ on the thermal parameter $x$. Also plotted in Figures 1 and 2 are the entropic and Bures degrees of nonGaussianity, Eqs. (3.4) and (3.5), respectively. Here we have performed numerically the corresponding summations making use of the probability distributions (2.3) and (3.2). As in the case of a PATS 9, non-Gaussianity of a PSTS increases with the number of
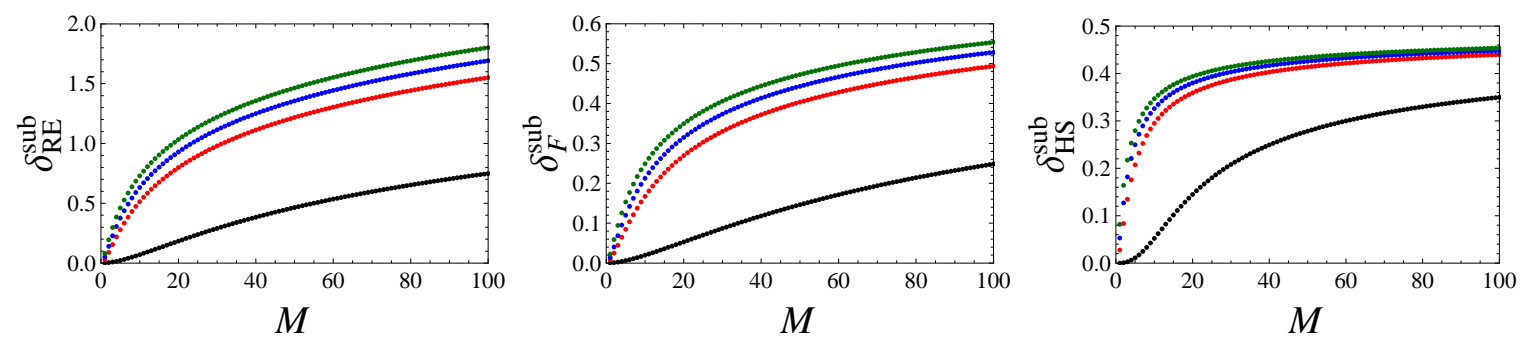

Figure 1. Dependence of the distance-type measures of non-Gaussianity on the number of subtracted photons. All the plots start from origin. The lowest curve is for $\bar{n}=0.1$. For the upper ones we have used $\bar{n}=1,2,5$, respectively.

subtracted photons. As one can see from Figure 1 of Ref. [9], when adding photons to a thermal state, non-Gaussianity decreases with the thermal parameter $x$, which is not the case for a PSTS in our present Figure 2. We have to remark that the three 

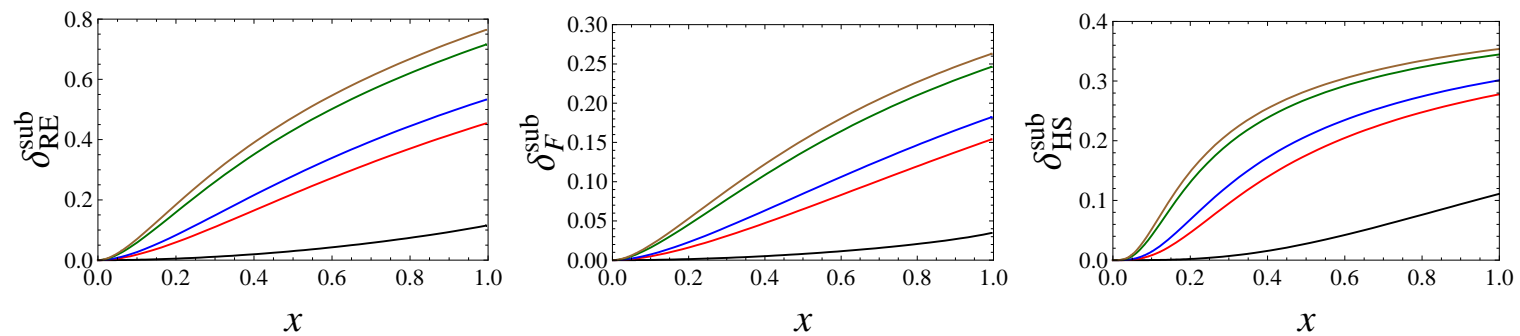

Figure 2. Dependence of the distance-type measures of non-Gaussianity on the thermal parameter $x$ for $M$-photon-subtracted thermal states. All the plots start from origin. The lowest plot is for $M=1$. For the upper ones we have used $M=4,5,8,9$, respectively.

measures we used here give similar dependencies on the parameters involved, which is a signature of their consistency. On physical grounds, we expect that a good measure of non-Gaussianity has a monotonic behaviour with respect to the mean photon number $\langle\hat{n}\rangle$ and, in turn, to the parameters entering its expression (2.6). According to our plots in Figures 1 and 2, this is verified for the class of PSTSs for all the measures investigated here.

\section{Gaussification by damping}

In our paper [10] the evolution under the quantum optical master equation of a Fockdiagonal density matrix was conveniently written in the interaction picture:

$$
\begin{aligned}
& \rho_{j k}(t)=\delta_{j k} \frac{\left[\bar{n}_{T}(t)\right]^{j}}{\left[\bar{n}_{T}(t)+1\right]^{j+1}} \sum_{l=0}^{\infty} \rho_{l l}(0)\left[\frac{\left(\bar{n}_{R}+1\right)\left(1-e^{-\gamma t}\right)}{\bar{n}_{T}(t)+1}\right]^{l} \\
& \times{ }_{2} F_{1}\left[-j,-l ; 1 ; \frac{e^{-\gamma t}}{\left(\bar{n}_{R}+1\right)\left(1-e^{-\gamma t}\right) \bar{n}_{T}(t)}\right] .
\end{aligned}
$$

In Eq. (4.1), $\bar{n}_{R}$ and $\gamma$ are constants of the thermal bath and $\bar{n}_{T}(t):=\bar{n}_{R}\left(1-e^{-\gamma t}\right)$. The limit $t \rightarrow \infty$ in Eq. (4.1) represents a thermal state with the Bose-Einstein mean photon occupancy $\bar{n}_{R}$. We thus deal with an evolving Gaussification process which eventually destroys both the non-Gaussianity and the non-classicality properties of any input state. The corresponding time-dependent associate Gaussian state is a thermal one whose mean occupancy is equal to the average photon number of the damped field state. We find:

$$
\left.\langle\hat{n}\rangle\right|_{t}=[\bar{n}(M+1)] e^{-\gamma t}+\bar{n}_{T}(t) .
$$

By employing Eqs. (A.2) and (A.4) we obtain the following expression of the photonnumber distribution in a damped PSTS:

$$
\begin{aligned}
& p_{n}^{\mathrm{sub}}(t)=\left.\left[\hat{\rho}_{M}^{\mathrm{sub}}(\bar{n})\right]_{n n}\right|_{t}=\frac{\left[\bar{n}_{T}(t)+1\right]^{M}\left[\bar{n} \mathrm{e}^{-\gamma t}+\bar{n}_{T}(t)\right]^{n}}{\left[\bar{n} \mathrm{e}^{-\gamma t}+\bar{n}_{T}(t)+1\right]^{M+n+1}} \\
& \quad \times{ }_{2} F_{1}\left(-M,-n ; 1 ; \frac{\bar{n} \mathrm{e}^{-\gamma t}}{\left[\bar{n}_{T}(t)+1\right]\left[\bar{n} \mathrm{e}^{-\gamma t}+\bar{n}_{T}(t)\right]}\right) .
\end{aligned}
$$


We have used the time-dependent probability distribution (4.3) and the mean photon occupancy (4.2) to evaluate numerically two of the distance-type measures of nonGaussianity we are interested in: $\left.\delta_{\mathrm{RE}}\left[\hat{\rho}_{M}^{\mathrm{sub}}(\bar{n})\right]\right|_{t}$ and $\left.\delta_{\mathrm{F}}\left[\hat{\rho}_{M}^{\mathrm{sub}}(\bar{n})\right]\right|_{t}$ via Eqs. (3.4) and (3.5), respectively. The last degree of non-Gaussianity we consider here is the HilbertSchmidt one, Eq. (3.3). As in the case of $M$-photon added thermal states [10, the necessary ingredients to evaluate the Hilbert-Schmidt degree of non-Gaussianity can be obtained analytically. However, we do not write here the explicit expressions of the time-dependent purity and generating function. Our results are displayed in Figure 3 , where the time evolutions of the three non-Gaussianity measures are presented for the same values of the parameters. We now take advantage of our previous results
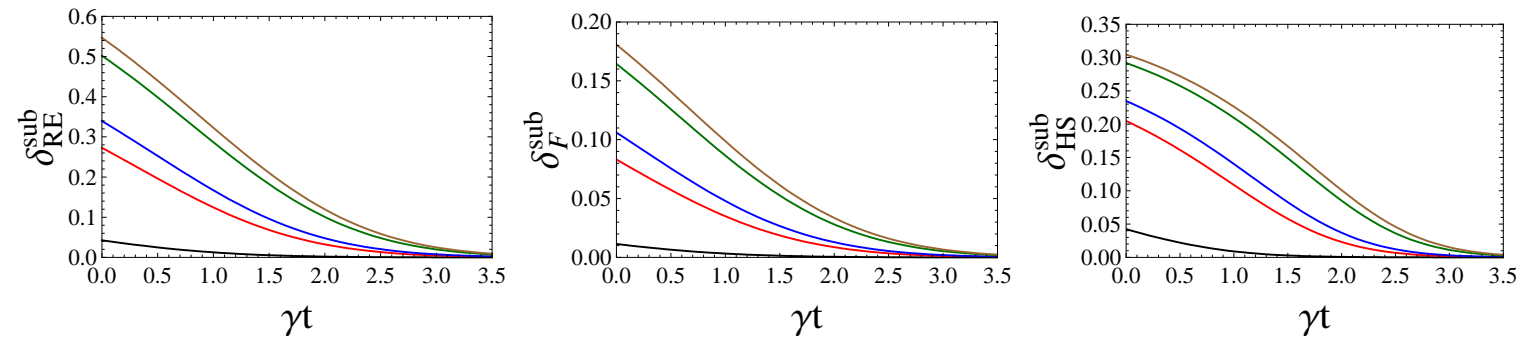

Figure 3. Time evolution of non-Gaussianity for $M$-photon-subtracted thermal states. We used the following parameters: $\bar{n}=1.5, \bar{n}_{R}=0.1, M=1,4,5,8,9$. The upper plots correspond to the higher values of $M$.

on the non-Gausssianity of damped PATSs [10]. In Figure 4 we present a comparison between the time evolution of non-Gaussianity for photon-added (dotted curves) and photon-subtracted (continuous curves) corresponding to the same parameters of states and reservoir.
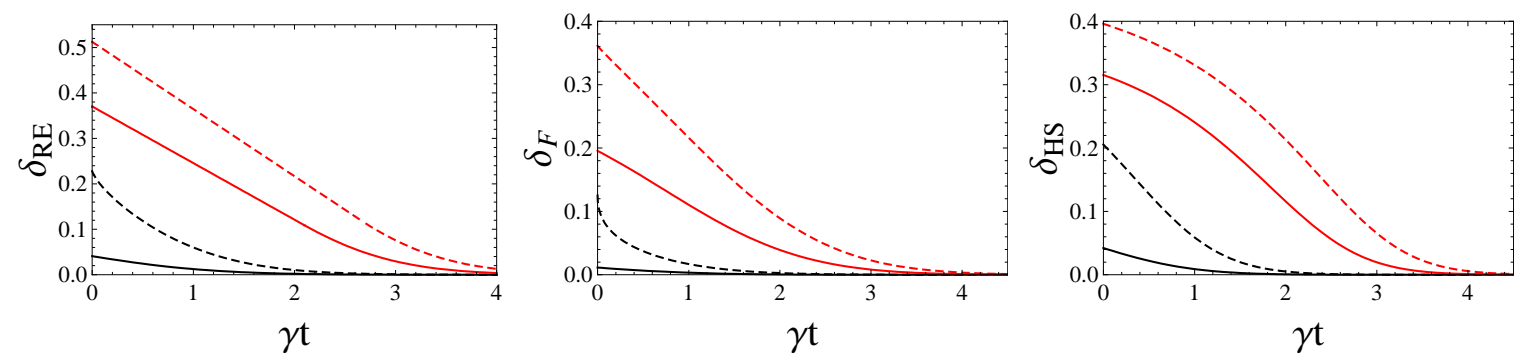

Figure 4. Time evolution of non-Gaussianity for $M$-photon-subtracted thermal states (continuous lines) and $M$-photon-added thermal states (dottted lines). We used the following parameters: $\bar{n}=1.5, \bar{n}_{R}=0.1, M=1$ (lower plots) and $M=10$ (upper plots).

\section{Conclusions}

Non-Gaussianity of pure states is often associated to their non-classicality. In this work we have examined the class of photon-subtracted one-mode thermal states, which are 
always mixed and classical. Subtraction of $M$ photons from a thermal state results in a photon-number distribution which turns out to be a negative binomial one whose stopping number is equal to $M+1$. The principal feature we have looked for was the non-Gaussianity of the PSTSs, as indicated by some recently introduced distance-type measures. Also investigated was the decrease of this property during the interaction of the field mode with a thermal reservoir described by the quantum optical master equation. We have shown that decaying of non-Gaussianity was consistently pointed out by three distance-type measures. Thus, the fidelity-based degree, the Hilbert-Schmidt one, and the entropic measure evolve monotonically, as expected for any good measure of non-Gaussianity [9, 10]. Moreover, we have compared this evolution to that of the photon-added thermal states which, by contrast, are non-classical. We have found that a given PATS has a larger amount of non-Gaussianity than the corresponding PSTS. This inequality between the degrees of non-Gaussianity of PATSs and PSTSs is maintained during their dissipative evolution. We conclude by stressing the significance of the agreement between the three measures of non-Gaussianity employed here, which is equally valid for both the PATSs and the PSTSs. Because the entropic measure $\delta_{\mathrm{RE}}[\hat{\rho}]$ is an exact one [11], the other two, $\delta_{\mathrm{F}}[\hat{\rho}]$ and $\delta_{\mathrm{HS}}[\hat{\rho}]$, albeit approximate, are nevertheless reliable.

\section{Acknowledgments}

This work was supported by the Romanian National Authority for Scientific Research through Grant No. PN-II-ID- PCE-2011-3-1012 for the University of Bucharest.

\section{Appendix A. Some useful formulae involving Gauss hypergeometric functions}

A Gauss hypergeometric function is the sum of the corresponding hypergeometric series:

$$
{ }_{2} F_{1}(a, b ; c ; z):=\sum_{n=0}^{\infty} \frac{(a)_{n}(b)_{n}}{(c)_{n}} \frac{z^{n}}{n !}, \quad(|z|<1),
$$

where $(a)_{n}:=\Gamma(a+n) / \Gamma(a)$ is Pochhammer's symbol standing for a rising factorial. This definition is extended by analytic continuation [30]. Recall Pfaff's transformation formula [30],

$$
{ }_{2} F_{1}(a, b ; c ; z)=(1-z)^{-b} F_{1}\left(c-a, b ; c ; \frac{z}{z-1}\right),
$$

as well as Murphy's expression of the Legendre polynomial of degree $l$ in terms of a Gauss hypergeometric function [30]:

$$
\mathcal{P}_{l}(z)={ }_{2} F_{1}\left(-l, l+1 ; 1 ; \frac{1-z}{2}\right), \quad(l=0,1,2,3, \ldots) .
$$


The following sum [30] has been used for obtaining the photon-number distribution (4.3) in a damped PSTS:

$$
\sum_{n=0}^{\infty} \frac{(-\xi)_{n}}{n !}(-t)^{n}{ }_{2} F_{1}(-n, b ; c ; z)=(1+t)^{\xi}{ }_{2} F_{1}\left(-\xi, b ; c ; \frac{t z}{1+t}\right) .
$$

\section{References}

[1] Dodonov V V 2002 J. Opt. B: Quantum Semiclass. Opt. 4 R1

[2] Dakna M, Knöll L, and Welsch D-G 1998 Eur. Phys. J D3 295

[3] T. Opatrný, G. Kurizki, and D.-G. Welsch, Phys. Rev. A 61, 032302 (2000).

[4] A. Mari and J. Eisert, Phys. Rev. Lett. 109, 230503 (2012).

[5] V. Veitch, N. Wiebe, C. Ferrie, and J. Emerson, New J. Phys. 15, 013037 (2013).

[6] Genoni M G, Paris M G A, and Banaszek K 2007 Phys. Rev. A 76042327

[7] Genoni M G, Paris M G A, and Banaszek K 2008 Phys. Rev. A 78060303

[8] Genoni M G and Paris M G A 2010 Phys. Rev. A 82052341

[9] Ghiu I, Marian P, and Marian T A 2013 Phys. Scr. T153 014028

[10] Marian P, Ghiu I, and Marian T A 2013 Phys. Rev. A 88012316

[11] Marian P and Marian T A 2013 Phys. Rev. A 88012322

[12] Barbieri M, Spagnolo N, Genoni M G, Ferreyrol F, Blandino R, Paris M G A, Grangier P, and Tualle-Brouri R 2010 Phys. Rev. A 82063833

[13] Allevi A, Andreoni A, Bondani M, Genoni M G, and Olivares S 2010 Phys. Rev. A 82013816

[14] Allevi A, Olivares S, and Bondani M 2012 Opt. Express 2024850

[15] Allevi A, Bondani M, Marian P, Marian T A, and Olivares S 2013 J. Opt. Soc. Am. B 302621

[16] Agarwal G S and Tara K 1991 Phys. Rev. A 43492

[17] Zhang Z and Fan H 1992 Phys. Lett. A 16514

[18] Dodonov V V, Korennoy Y A, Manko V I and Moukhin Y A 1996 Quant. Semicl. Opt. 8413

[19] Mizrahi S S and Dodonov V V 2002 J. Phys. A 358847

[20] Wenger J, Tualle-Brouri R and Grangier P 2004 Phys. Rev. Lett. 92153601

[21] Ourjoumtsev A, Tualle-Brouri R, Laurat J and Grangier P 2006 Science 31283

[22] Parigi V, Zavatta A, Kim M S and Bellini M 2007 Science 3171890

[23] Zavatta A, Parigi V, Kim M S, and Bellini M 2008 New Journal of Physics10 123006

[24] Kim M S, Park E, Knight P L, and Jeong H 2005 Phys. Rev. A 71043805

[25] Biswas A and Agarwal G S 2007 Phys. Rev. A 75032104

[26] Wang S, Hu L Y, and Fan H Y 2012 Eur. Phys. J D 66 art.166

[27] Kim M S 2008 J. Phys. B 41133001

[28] Zhai Y et al. 2013 Opt. Expr. 382171

[29] Feller W 1970 An Introduction to Probability Theory and Its Applications vol 1, Third Edition (New York: John Wiley \& Sons). See pp. 164-166

[30] Erdélyi A, Magnus W, Oberhettinger F and Tricomi F G 1953 Higher Transcendental Functions vol 1 (New York: McGraw-Hill) 